\title{
Implementación del Convenio Marco de la Organización Mundial de la Salud para el Control del Tabaco y la Prevención del Cáncer en Colombia
}

\section{Implementation in Colombia of the Framework Convention on Tobacco Control to Prevent Cancer: Achievements and Challenges \\ Implementação do Convenção-Quadro para o Controle do Tabaco e a prevenção do cancro na Colômbia}

\author{
Luis Miguel Tellez Neira, $\mathrm{Mg}^{1 *}$ \\ Sandra Romero-Torres ${ }^{1}$ \\ Maria Isabel Calderón Cortés ${ }^{1}$
}

Recibido: 30 de abril de 2019 - Aceptado: $1^{\circ}$ de octubre de 2019

Doi: https://doi.org/10.12804/revistas.urosario.edu.co/revsalud/a.8780

Para citar este artículo: Tellez Neira LM, Romero-Torres S, Calderón Cortés MI. Implementación del Convenio Marco de la Organización Mundial de la Salud para el control del tabaco y la prevención del cáncer en Colombia. Rev Cienc Salud. 2020;18(1):169-187. https://doi. org/10.12804/revistas.urosario.edu.co/revsalud/a.8780

\section{Resumen}

Introducción: el tabaquismo es un problema global. Un problema tan importante que hizo que la Organización Mundial de la Salud liderara el Convenio Marco Para el Control del Tabaco, del cual son parte la gran mayoría de países del mundo. En ese contexto, Colombia ha realizado varias acciones para reducir la prevalencia del tabaquismo; estas acciones están enmarcadas en el Plan Decenal para el Control del Cáncer 2012-2021. Desarrollo: se analizó la implementación de las acciones planteadas por el Plan Decenal para el Control del Cáncer y su correlación con el Convenio Marco de la Organización Mundial de la Salud, bajo la implementación del Plan de Medidas MPowER. Conclusiones: Colombia ha implementado varias estrategias para el control del tabaco, enmarcadas en el Plan Decenal para el Control del Cáncer. Entre estas estrategias, se encuentra un sistema de vigilancia del consumo de tabaco, políticas de control, espacios cien por ciento libres de humo de tabaco, el Programa para la Cesación del Consumo de Tabaco y Atención 
del Tabaquismo, advertencias sanitarias moderadas, campañas de comunicación, prohibición completa de publicidad, promoción y patrocinio, así como un aumento de los impuestos al tabaco. A pesar de estas implementaciones, el reto actual es intervenir aquellas medidas en las que menos se ha trabajado o que han tenido menor resultado para lograr que el control del tabaquismo sea más efectivo; también es necesario hacer frente al uso de cigarrillos electrónicos. Las medidas descritas deberían implementarse sin la interferencia de la industria tabacalera.

Palabras clave: tabaco; Convenio Marco de la oms para el Control del Tabaco; neoplasias; Colombia; política de salud.

\section{Abstract}

Introduction: Smoking is a global issue that motived the World Health Organization to lead the Framework Convention on Tobacco, adopted by many countries. In that context, Colombia has carried out some actions to reduce smoking prevalence, taking into account the Ten-Year Plan for Cancer Control 20122021. Development: This article analyzes the implementation of the actions proposed by the Ten-Year Plan for Cancer Control 2012-2021 and its correlation with the Framework Convention on Tobacco's MPOWER Package. Conclusion: Colombia has implemented some strategies to control smoking through the Ten-Year Plan for Cancer Control 2012-2021, such as the smoking monitoring system, control policies, smoke-free areas, the National Program for Smoking Cessation, moderate health warnings, communication campaigns, total restriction to any kind of advertisement and promotion, and raising tax of tobacco products. In spite of those achievements, it is necessary to strengthen the weakest strategies to reduce smoking, as well as facing electronic cigarette use. These actions must be carried out without tobacco industry interference.

Keywords: tobacco; wHo Framework Convention on tobacco; neoplasms; Colombia; health policies.

\section{Resumo}

Introdução: o tabaquismo é um problema global que orientou à Organização Mundial da Saúde a liderar o Convenção-Quadro para o Controle do Tabaco, do qual são parte a grande maioria de países. Nesse contexto, a Colômbia tem realizado várias ações para reduzir a prevalência do tabaquismo enquadradas no Plano Decenal para o Controle do Cancro 2012-2021. Desenvolvimento: se analisa a implementação das ações apresentadas pelo Plano Decenal para o Controle do Cancro e sua correlação com a ConvençãoQuadro sob a implementação do Plano de Medidad MPower. Conclusões: a Colômbia tem implementado várias estratégias para o controle do tabaco enquadradas no Plano Decenal para o Controle do Cancro entre as que se encontra o sistema de vigilância de consumo de tabaco, políticas de controle, espaços 100 \% livres de fumo de tabaco, o Programa Nacional para a Cessação, advertências sanitárias moderadas, campanhas de comunicação, proibição completa de publicidade, promoção e patrocínio e aumento dos impostos ao tabaco. Apesar do anterior, o desafio é intervir nas medidas com maior retrocesso para conseguir que o controle do tabaquismo seja mais efetivo; também é necessário enfrentar ao uso de cigarros eletrônicos. $\mathrm{O}$ anterior, sem interferência da indústria do tabaco.

Palavras-chave: tabaco; Convenção-Quadro para o Controle do Tabaco; neoplasias; Colômbia; política de saúde.

\section{Introducción}

$E_{\text {nivel mundial (1). En Colombia, se estima que el } 7.3 \% \text { de la población de los diez años de }}^{\text {l tabaquismo es un problema de salud pública que causa ocho millones de defunciones a }}$ 
edad en adelante consume tabaco y que 32000 personas fallecen al año debido a esta actividad $(2,3)$. Por eso, en los últimos años se han tomado medidas al respecto, orientadas por el Convenio Marco de la Organización Mundial de la Salud (oms) para el Control del Tabaco; se trata del primer tratado mundial en salud pública aceptado por la Unión Europea y por ciento ochenta países, entre ellos Colombia (estos países representan a más del $90 \%$ de la población mundial), comprometidos en la lucha contra el tabaquismo.

Además de haber aceptado el Convenio Marco de la oms para el Control del Tabaco, Colombia promulgó el Plan Decenal para el Control del Cáncer (PDCc) 2012-2021, que a su vez se enmarca en el Modelo para el Control del Cáncer, un “conjunto de actividades que de forma organizada se orienta a la disminución de la carga de cáncer en Colombia, mediante la reducción de los factores de riesgo de los diferentes tipos de cáncer, para así disminuir la mortalidad y aumentar la calidad de vida de quienes padecen esta enfermedad” (5 p.7) (4). Ese modelo propone acciones con base en tres herramientas (comunicación, educación y movilización social), que se implementan en tres ámbitos (políticas públicas, espacios comunitarios y servicios de salud) para lograr las líneas estratégicas planteadas en el Plan Decenal para el Control del Cáncer (control del riesgo, detección temprana, tratamiento y rehabilitación, y cuidado paliativo). Específicamente la reducción del tabaquismo es una acción enmarcada en la primera línea señalada por el modelo (control del riesgo).

El objetivo de este artículo es analizar las acciones planteadas por el PDCc 2012-2021 para la reducción del tabaquismo y su correlación con las disposiciones dictadas por el Convenio Marco de la oms para el Control del Tabaco. De manera específica, se identifican las acciones señaladas en el PDCc 2012-2021, cuya ejecución se ha llevado a cabo en los tres ámbitos del modelo (políticas públicas, espacios comunitarios y servicios de salud) relacionados con el Plan de Medidas MPOWER (una herramienta diseñada por la oms para facilitar la implementación del Convenio en cada país (tabla 1), así como algunos avances alcanzados y retos por superar frente al control del tabaquismo (6).

Tabla 1. Componentes del Plan de Medidas MPOWER

\begin{tabular}{|c|c|c|}
\hline Monitor & $\begin{array}{l}\text { Vigilar el consumo de } \\
\text { tabaco y las políticas } \\
\text { de prevención }\end{array}$ & $\begin{array}{l}\text { Acciones para establecer sistemas de vigilancia que brinden información } \\
\text { sobre indicadores en prevalencia del consumo de tabaco, repercusiones } \\
\text { de las intervenciones normativas y promoción y cabildeo de la industria } \\
\text { tabacalera. }\end{array}$ \\
\hline Protect & $\begin{array}{l}\text { Proteger a la pobla- } \\
\text { ción del humo de ta- } \\
\text { baco }\end{array}$ & $\begin{array}{l}\text { Promoción e implementación de ambientes } 100 \% \text { libres de humo de taba- } \\
\text { co, los cuales permiten la protección de la población del humo de segunda } \\
\text { mano y ayudan a reducir el consumo de tabaco. }\end{array}$ \\
\hline Offer & $\begin{array}{l}\text { Ofrecer ayuda para el } \\
\text { abandono de tabaco }\end{array}$ & $\begin{array}{l}\text { Métodos de tratamiento para tratar la dependencia al tabaco a cargo de los } \\
\text { sistemas de atención sanitaria de los países. La oms recomienda tres tipos } \\
\text { de tratamiento: 1) asesoría sobre abandono en los servicios de atención } \\
\text { primaria de los sistemas de salud; } 2 \text { ) líneas telefónicas de apoyo accesibles } \\
\text { y; 3) acceso a farmacoterapia de bajo costo. } \\
\text { (Continúa) }\end{array}$ \\
\hline
\end{tabular}




\begin{tabular}{ccc}
\hline Warning & $\begin{array}{c}\text { Advertir acera de los } \\
\text { peligros del tabaco }\end{array}$ & $\begin{array}{c}\text { Reglamentación del uso de advertencias sanitarias en los envases de todos } \\
\text { los productos de tabaco por medio de imágenes de gran tamaño, legibles y } \\
\text { con mensajes de alerta. También, inclusión de campañas de publicidad que } \\
\text { resalten los peligros asociados, los beneficios del abandono del tabaco y la } \\
\text { protección frente al humo de segunda mano. }\end{array}$ \\
\hline Enforce & $\begin{array}{c}\text { Hacer cumplir las } \\
\text { prohibiciones sobre } \\
\text { publicidad, promo- } \\
\text { ción y patrocinio }\end{array}$ & $\begin{array}{c}\text { Prohibiciones frente a las acciones de promoción, patrocinio y mercadeo } \\
\text { realizadas por la industria tabacalera. }\end{array}$ \\
\hline Raise & $\begin{array}{c}\text { Aumentar los impues- } \\
\text { tos al tabaco }\end{array}$ & $\begin{array}{c}\text { Aumentar los precios del tabaco al incrementar los impuestos, lo cual es } \\
\text { considerado por la oms como la medida individual más eficaz para reducir } \\
\text { el consumo y promover la cesación. }\end{array}$ \\
\hline
\end{tabular}

Fuente: elaboración propia con información de la Organización Mundial de la Salud (OMS) (6).

Para este análisis, se realizó una revisión de la literatura relacionada con el control del tabaco, relacionada con los resultados de avance reportados por la oms y el pDcc 2012-2021.

Finalmente, también se hace una reflexión sobre la creciente acogida de los sistemas electrónicos de administración de nicotina y los sistemas similares sin nicotina (sEAN/sssN), conocidos comúnmente como "cigarrillos electrónicos". Si bien esos dispositivos no son considerados por el PDCC 2012-2021, su uso representa un problema creciente debido a que la industria tabacalera los diseña y promociona como métodos de cesación tabáquica, a pesar de no tener evidencia científica disponible que sustente ese argumento.

\section{Desarrollo}

\section{Implementación del Convenio Marco de la oms para el Control del Tabaco y su relación con el Plan Decenal para el Control del Cáncer (PDCC) 2012-2021}

Colombia promulgó el Plan Decenal para el Control del Cáncer (PDCC) 2012-2021 como principal respuesta de política pública para hacer frente a esa enfermedad. Este plan dicta varias disposiciones para el control del tabaco, las cuales se implementan en tres ámbitos: políticas públicas, espacios comunitarios y servicios de salud.

\section{Ámbito de políticas públicas}

En este ámbito se destaca la implementación de las leyes 1109 de 2006, 1335 de 2009 y 1819 de 2016 (7-9). Asimismo, es importante la resolución 1309 de 2012 ("Por la cual se crea el Comité de Etiquetado y Empaquetado de productos del tabaco y sus derivados”) y las acciones de vigilancia de consumo de tabaco. 
La Ley 1109 de 2006 es importante porque incorpora a la normatividad nacional el Convenio Marco de la oms para el Control del Tabaco, de modo que se convierte en un mandato cuyo cumplimiento es llevado a cabo por las demás leyes y acciones descritas en este artículo.

Es especialmente importante la Ley 1335 de 2009 (Ley Antitabaco), la cual responde a varias recomendaciones del Plan de Medidas MPowEr. Por ejemplo, el artículo 13 (reglamentado por la Resolución 3961 de 2009 del Ministerio de Salud y Protección Social) responde al componente relacionado con advertir acera de los peligros del tabaco (W: Warning), ya que ordena el uso de advertencias sanitarias en las etiquetas de todos los productos de tabaco con un tamaño mínimo del $30 \%$ del área de las dos caras principales de cada empaque. Además, la implementación de ese artículo está reglamentada por la Resolución 1309 de 2012, la cual establece la creación del Comité de Etiquetado y Empaquetado de productos del tabaco y sus derivados para asesorar a la Dirección de Promoción y Prevención del Ministerio de Salud y Protección Social en el trámite y aprobación o rechazo de las solicitudes de las simulaciones de productos de tabaco.

Un estudio del Instituto de Evaluación de Tecnologías Sanitarias (IETs, Colombia) y del Instituto de Efectividad Clínica y Sanitaria (IECS, Argentina) señala los beneficios logrados por el uso de advertencias sanitarias, tal como están ordenadas en el artículo 13 de la Ley 1335 de 2009, así como los beneficios adicionales que se lograrían si esas advertencias fueran más grandes. Allí se calcula que, gracias a las advertencias sanitarias reglamentadas por la Ley 1335 de 2009, a lo largo de diez años se evitarían 3465 muertes, 15779 eventos cardiovasculares y cerebro vasculares, 5657 casos de enfermedad pulmonar obstructiva crónica (ЕРОс) y 1339 de cáncer; además, se ahorrarían cop 538745 millones en costos del sistema de salud (10). Sin embargo, la reglamentación de la venta de productos de tabaco en empaquetado neutro (cajas que no usan logotipos, imágenes de marca o información promocional, que no correspondan al nombre comercial o al nombre del producto en un color, además del uso de un tipo de letra corriente) con advertencias sanitarias fuertes (aquellas cuyo tamaño cubre mínimo el 50\% de cada cara principal de cada empaque) permitiría, a lo largo de diez años, evitar 24086 muertes y 109675 eventos cardiovasculares y cerebro vasculares, 39321 casos de enfermedad pulmonar obstructiva crónica y 9304 de cáncer (10). La información anterior sugiere que el Estado colombiano debe reglamentar tanto el empaquetado neutro como las advertencias sanitarias fuertes, para ello se deben tener en cuenta las recomendaciones que la oms (11) brinda al respecto:

1. Utilizar el empaquetado neutro en todas las categorías de productos de tabaco.

2. Asegurar que los nombres de marca y de producto expuestos en un color y tipo de letra estándar sean la única forma de logotipo, color, imagen de marca o información promocional que figure en el empaquetado. 
3. Abordar en la legislación relativa al empaquetado neutro rasgos de diseño que hacen más atractivos los productos de tabaco.

4. Normalizar el aspecto de los productos de tabaco.

5. Evitar que etiquetas, estuches o cualquier tipo de elemento gráfico oculten o debiliten las advertencias sanitarias y otros mensajes apropiados.

6. Fijar un plazo máximo para que los fabricantes y los importadores preparen la impresión de los nuevos envases.

El Plan de Medidas MPOWER también resalta la necesidad de hacer vigilancia del consumo de tabaco (M: Monitor). Esta vigilancia se ha hecho concreta en varias encuestas como la Encuesta Nacional de Calidad de Vida 2016 y 2017 (2, 12), la Encuesta Nacional de Salud (ENs) 2007 (13) y la Encuesta Nacional de Consumo de Tabaco en Jóvenes, realizada en 2016 (14). También se destacan el Estudio Nacional de Consumo de Sustancias Psicoactivas 2008, 2013 y 2016 (15-17) y el Estudio de Consumo de Sustancias Psicoactivas en Bogotá 2009 y 2016 (18, 19) porque brindan más información sobre el consumo de tabaco en términos de prevalencia, incidencia, edad de inicio y percepción del riesgo, principalmente.

Aunque los datos generados en esas encuestas son importantes, es necesario contar con información estandarizada que permita observar el avance del país en lo que respecta a la reducción del consumo de tabaco en los diferentes grupos poblacionales. También es preciso indagar sobre otras dimensiones de la prevención del tabaquismo, como el uso de sistemas electrónicos de administración de nicotina, exposición al humo de tabaco de segunda mano, publicidad de la industria tabacalera y en contra del consumo de tabaco y conocimiento y actitudes de la población fumadora y no fumadora. Aunque la Encuesta Mundial de Tabaquismo en Jóvenes (ЕMTAJ) de la oms ya indaga sobre estos temas, es importante generar información sobre esas temáticas para la población adulta.

Además de los artículos antes mencionados parte de la Ley 1335 de 2009, también son importantes los artículos 347 y 348 de la Ley 1819 de 2016 (Reforma Tributaria Estructural), ya que gravan con impuestos (R: Raise) la venta de cigarrillos, tabacos, cigarros y cigarritos. Así, por ejemplo, se gravaron con cop1400 en 2017 y cop 2100 desde 2018 cada cajetilla de veinte unidades, mientras que los impuestos por cada gramo de picadura, rapé o chimú pasaron de cop 90 en 2017 a соP 167 desde 2018. Además, se adiciona proporcionalmente un componente ad valorem, equivalente al $10 \%$ de la base gravable por cada cajetilla de veinte unidades.

Un estudio realizado por el Banco Mundial estima que la reforma tributaria de 2016 evitaría, a lo largo de setenta y cinco años aproximadamente, 337300 muertes prematuras asociadas con el tabaquismo, 5140 casos de gastos por enfermedades catastróficas relacionadas y un ahorro para el Gobierno Nacional de aproximadamente cop 3.096 billones por atención a pacientes (un monto equivalente a aproximadamente el $7 \%$ del gasto nacional 
en salud o casi el 0.4\% del PIB en 2016); además, los ingresos fiscales anuales adicionales recaudados ascenderían a cerca del $3 \%$ del gasto total en salud de Colombia a corto plazo, y los quintiles más pobres sufrirían la menor carga fiscal. Por otro lado, Maldonado et al. desmienten un posible aumento significativo del contrabando de cigarrillos argumentado a favor de la industria tabacalera en el rechazo de esa medida pues los autores calcularon el tamaño del contrabando de cigarrillos en Bogotá, Medellín, Cali, Cartagena y Cúcuta (las cuales en conjunto representan el $63 \%$ del mercado de cigarrillos en Colombia) (20, 21). Así, después de nueve meses de entrar en rigor los artículos 347 y 348 de la Ley 1819 de 2016, los resultados indicaron que solo el $3.5 \%$ del consumo de cigarrillos en esas ciudades es de contrabando, aunque entre ellas hay grandes diferencias (por ejemplo, en Bogotá alcanzó el $1.5 \%$, mientras que en Cúcuta fue del $22.8 \%$ ).

Aumentar los impuestos al tabaco ha generado efectos positivos en varios países. Por ejemplo, Schafferer et al. estiman que en la Unión Europea el consumo de cigarrillos disminuiría aproximadamente un 3.1\% como resultado del aumento del $10 \%$ su precio, y en algunos países como la República Checa, Letonia, Lituania, Polonia y Eslovaquia, esa reducción sería cercana al 8\% (22). Teniendo en cuenta lo anterior, los impuestos sobre el tabaco se han introducido o se están introduciendo en diversos países como Botswana, Chile, Ecuador, India, México, Nigeria, Perú, Arabia Saudita, Sudáfrica, los Emiratos Árabes Unidos y el Reino Unido, ya que son reconocidos por la oms como "Best Buys” para prevenir y controlar no solo el cáncer, sino todas las enfermedades no transmisibles. Además, los impuestos son útiles para recaudar recursos; por ejemplo, la Ley de promoción de la salud de Tailandia de 2001 estableció un impuesto sobre el tabaco y el alcohol que permite recaudar aproximadamente usD 120 millones anuales para los esfuerzos de promoción de la salud doméstica. En la misma línea, en 2012, Filipinas aumentó los impuestos al tabaco y al alcohol y está utilizando los ingresos para complementar los esfuerzos para lograr la cobertura universal de salud; después de tres años de implementación, se recolectaron usD 3.9 mil millones en ingresos adicionales, el $80 \%$ de los cuales se usaron para financiar la extensión del seguro de salud al $40 \%$ de la población más pobre (23).

Los estudios señalados muestran que Colombia debe tomar medidas adicionales para elevar aún más el precio de los productos de tabaco, ya que este aún es bajo en comparación con otros países de ingresos medios y frente a algunos de América Latina (24) (tabla 2). 
Tabla 2. Precio en dólares por cada caja de cigarrillos 2016 ajustados por paridad de poder adquisitivo

\begin{tabular}{ccc}
\hline Ingresoso & País & Precio en dólares \\
\hline & India & 9.2 \\
& Indonesia & 5.2 \\
Países de ingresos & Bangladesh & 3.4 \\
medios-altos & Filipinas & 2.3 \\
& Vietnam & 2.6 \\
& Armenia & 3.1 \\
\hline & China & 2.8 \\
& México & 5.7 \\
Países de ingresos & Turquía & 10.3 \\
medios-bajos & Brasil & 3.2 \\
& Colombia & 2.2 \\
& Tailandia & 7.1 \\
& Chile & 5.8 \\
\hline
\end{tabular}

Fuente: Global Tobacco Economics Consortium (24).

Las medidas impositivas inducen al abandono del hábito tabáquico, evitan su inicio, reducen el número de recaídas entre quienes han dejado de fumar y disminuyen el consumo entre quienes siguen fumando. La oms indica que un aumento del precio de un $10 \%$ por paquete de cigarrillos debería generar una reducción de la demanda equivalente a aproximadamente un 4\% en los países de ingresos altos, y a entre un $4 \%$ y un $8 \%$ en los países de ingresos bajos y medios, en donde la población es más sensible a cambios de precios. Estas medidas también pueden influir el consumo en niños y adolescentes, quienes son más sensibles a las subidas de precios que los adultos (25).

Sin embargo, también se debe aprobar una ley que incorpore el Protocolo para la Eliminación del Comercio Ilícito de Productos de Tabaco ${ }^{2}$ al ordenamiento jurídico nacional, tal como ya lo han hecho Ecuador, Nicaragua, Uruguay, Brasil, Costa Rica y Panamá (26). La aprobación de este paquete (el cual constituye un tratado internacional en sí mismo) es importante teniendo en cuenta que usualmente la industria tabacalera se opone al aumento de impuestos argumentando que aumentará el contrabando.

2 El Protocolo para la Eliminación del Comercio Ilícito de Productos de Tabaco es un grupo de medidas de alcance global para dar respuesta a los riesgos asociados con la compra y la venta ilegal de esos productos. Este Protocolo ha sido adoptado por la Conferencia de las Partes, entre las cuales está la Unión Europea y aquellos países que hacen parte del convenio. 


\section{Ámbito comunitario}

El pDcc 2012-2021 también plantea el desarrollo de intervenciones poblacionales y estrategias de movilización social para la veeduría ciudadana y el cumplimiento de la reglamentación de los ambientes $100 \%$ libres de humo de tabaco; la ejecución de estrategias educativas, así como el fortalecimiento de estrategias de comunicación masiva (las acciones de comunicación y educación concuerdan con el artículo 12 del Convenio "Educación, comunicación, formación y concientización del público”); y la generación de alianzas para desincentivar el consumo y la exposición al humo de tabaco y sus derivados.

Al respecto, los artículos 14 al 17 de la Ley 1335 de 2009 prohíben todas las formas de promoción y patrocinio de productos de tabaco (E: Enforce) en eventos culturales y deportivos; en medios de comunicación como radio, televisión o cine; en medios escritos como boletines, periódicos, revistas o cualquier documento de difusión masiva; en producciones teatrales $\mathrm{u}$ otras funciones en vivo; en funciones musicales en vivo o grabadas; $\mathrm{y}$ en video o filmes comerciales, discos compactos, discos de video digital o medios similares. Además, no se permite la fijación de vallas, pancartas, murales, afiches, carteles o similares móviles o fijos relacionados con la promoción del tabaco y sus derivados (estos artículos fueron declarados exequibles por la Corte Constitucional por medio de la Sentencia C-830/10 (27)). Se ha calculado (28) que el cumplimiento de esos artículos evitaría cerca de 10584 muertes, 48194 eventos cardiovasculares y cerebro-vasculares, 17279 casos de EPoc y 4088 de cáncer, y haría posible ahorrar cop1645 484 millones al sistema de salud. Sin embargo, vale la pena resaltar que las anteriores cifras corresponden al máximo acatamiento de las medidas del componente "E: Enforce” de MPOWER y que, en 2019, Colombia registró siete de diez puntos posibles en cuanto al cumplimiento de esas medidas (1).

Es necesario garantizar estas medidas orientadas a eliminar todas las formas de promoción y patrocinio de productos de tabaco. Al respecto, la Veeduría Ciudadana para el Control del Tabaco (29), una coalición de ciudadanos y organizaciones de la sociedad civil, se destaca como un actor que promueve la ejecución de acciones y políticas para la reducción del tabaquismo, que den cumplimiento al Convenio, y hagan frente a los intereses de la industria tabacalera. Aunque el rol de esa organización es clave, denunciar las estrategias desarrolladas por la industria tabacalera debe ser una prioridad de salud pública desde el Gobierno Nacional, lo cual no puede limitarse a la realización de acciones de inspección, vigilancia y control, sino que debe generar información estratégica periódica en este asunto.

Las prohibiciones mencionadas han sido complementadas con acciones de comunicación. Por ejemplo, el Ministerio de Salud y Protección Social ha liderado la campaña "Generación más: somos más sin tabaco") y el Instituto Nacional de Cancerología, "No fumar es la actitud”, que invitan a los niños y adolescentes a no fumar y que socializan los riesgos y consecuencias de fumar, de una manera creativa y en espacios frecuentados por esta población (30, 31). 
Es necesario realizar más estrategias similares, para ello se debe tener en cuenta que hay evidencia que indica que esas campañas de prevención son más efectivas cuando están dirigidas a poblaciones de preadolescente y de personas más jóvenes, y que su efectividad resulta afectada por las interacciones que una persona pueda tener con sus grupos sociales, por ejemplo, con su familia o con su entorno cultural (32). También es importante mencionar el trabajo de Goldman y Glantz, quienes revisaron la eficacia de los mensajes antitabaco transmitidos en los medios y condujeron un estudio cualitativo en algunos estados de Estados Unidos, centrado en 186 grupos focales, en el cual más de 1500 personas evaluaron dichos mensajes (33). Los autores encontraron que es particularmente efectivo incorporar en las campañas mensajes sobre los contenidos manipulativos de la industria tabacalera porque permite a los adolescentes concluir que su presunta decisión de fumar en realidad no es independiente, aunque las siguientes estrategias también son apropiadas para difundir mensajes antitabaco en medios masivos:

1. Mostrar los efectos del humo de segunda mano ente la población no fumadora.

2. Resaltar el carácter tóxico y altamente adictivo de los cigarrillos.

3. Advertir que dejar de fumar no es sencillo y que la mayoría de las personas lo logran después de muchos fracasos.

4. Exponer la facilidad con la cual los jóvenes pueden comprar productos de tabaco.

5. Resaltar los efectos a corto plazo (como dolores de cabeza y mal olor en la ropa) para que el tabaquismo deje de ser visto como algo atractivo.

6. Alertar sobre los efectos que el tabaquismo causa a largo plazo (especialmente enfermedades como el cáncer).

7. Señalar que la mayoría de las personas no fuman y que para ellas fumar es socialmente indeseable.

Estos hallazgos deben tenerse en cuenta por las instituciones que realicen campañas de comunicación antitabaco. También es importante realizar evaluaciones sobre las estrategias utilizadas para identificar las más exitosas, ya que, hasta la fecha, no es posible identificar si algunos mensajes o canales de comunicación son más efectivos que otros.

Adicionalmente, el artículo 19 de la Ley Antitabaco prohíbe el consumo de productos de tabaco en instituciones de salud, de educación, museos y bibliotecas, medios de transporte, establecimientos donde se atiende a menores de edad, entidades públicas y privadas destinadas a cualquier tipo de actividad (industrial, comercial o de servicios), áreas con alto riesgo de combustión (por ejemplo estaciones de gasolina) y espacios culturales y deportivos (P: Protect); mientras que el artículo 20 asigna obligaciones a los propietarios, empleadores y administradores de esos lugares para garantizar esas restricciones. Además, según el artículo 10, los gobernadores, los alcaldes y las secretarías departamentales, distritales y 
municipales de salud deben garantizar el cumplimiento de todas las disposiciones ordenadas por esa ley. Esas medidas se complementan a los artículos 38, 94 y 146 de la Ley 1801 de 2016 (Código Nacional de Policía y Convivencia), ya que establecen sanciones a quienes faciliten el consumo de tabaco a menores de edad, a quienes consuman tabaco en lugares no autorizados o a quienes lo hagan en sistemas de transporte público colectivo e individual de pasajeros (P: Protect) (34).

Existe evidencia que ayuda a calcular los beneficios asociados con la reglamentación de los ambientes $100 \%$ libres de humo de tabaco por medio de la normatividad mencionada. Un estudio del IETS y el IECS encuentra que esta reglamentación evitaría 18151 muertes, 82650 eventos cardiovasculares y cerebro-vasculares, 29632 casos de EPOc y 7011 de cáncer a lo largo de diez años y que, además, permitirán ahorrar cop 2821894 millones por costos al sistema de salud (35). Sin embargo, el reporte de 2019 de la oms sobre el Plan de Medidas MPOWER indica que Colombia registró siete de diez puntos posibles en cuanto al cumplimiento de esas medidas (1). Esto muestra que es necesario realizar esfuerzos adicionales para que el país pueda alcanzar todos los beneficios potenciales que la implementación de los ambientes $100 \%$ libres de humo de tabaco le puede proporcionar.

\section{Ámbito de servicios de salud}

En este ámbito, el PDcc 2012-2021 propone implementar guías de práctica clínica, programas de cesación basados en evidencia científica con cobertura nacional y capacitar a los profesionales de los servicios de la salud en estrategias de cesación y en temas de control del consumo y exposición al humo de tabaco y sus derivados. Todas estas medidas corresponden a la medida de ofrecer ayuda de MPOWER (O: Offer).

Al respecto, el Instituto Nacional de Cancerología publicó el libro Cesación tabáquica: Compilación de las recomendaciones actualizadas para profesionales de la salud en Colombia (36), en el que se brinda información sobre políticas de control, programas de cesación, resultados de investigaciones y recomendaciones para la cesación, eficacia y seguridad del uso de medicamentos, terapias alternativas e intervenciones de consejería, principalmente.

En la misma línea, desde 2017, el Ministerio de Salud y Protección Social lidera el Programa para la Cesación del Consumo de Tabaco y Atención del Tabaquismo, el cual está articulado alrededor de los siguientes seis componentes: primero, fortalecimiento del sistema de salud (para garantizar a todos los consumidores el acceso a intervenciones para la atención del tabaquismo); segundo, atención básica (realizadas en el marco de la consulta, con una duración de tres a diez minutos); tercero, atención intermedia (acciones breves y programadas en las instituciones prestadoras de servicios de salud de la red primaria en las cuales los fumadores reciben un plan de manejo y de seguimiento para lograr la abstinencia); cuarto, atención avanzada (tratamientos más complejos suministrados por especialidades interdisciplinarias, 
por ejemplo, intervenciones cognitivo-conductuales, entrenamiento en manejo de recaídas, intervenciones motivacionales y tratamientos farmacológicos); quinto, intervenciones comunitarias (aquellas que hacen uso de estrategias de información y comunicación, con énfasis en el autocuidado, en los grupos de apoyo y en los materiales de autoayuda); y, sexto, registro, seguimiento y monitoreo de las acciones mencionadas (37).

Debido a que aún no hay resultados sobre la implementación del Programa — seguramente porque fue recientemente puesto a disposición del público general—, el Ministerio de Salud y Protección Social debe asegurar que los prestadores de atención primaria desarrollen las capacidades necesarias para ofrecer las intervenciones allí mencionadas de manera sistemática, ya que la misma entidad reconoce que más del 50\% de los prestadores de atención primaria no ofrecen estas intervenciones sistemáticas de cesación, debido a la falta de conocimientos acerca del tabaco y su control, a la falta de definición de lineamientos y a la ausencia de capacidad con respecto a su prevención y tratamiento (37).

Lo realizado por el Ministerio de Salud y Protección Social y el Instituto de Nacional de Cancerología son una serie de lineamientos generales y recomendaciones que deben ser implementados por las instituciones en salud y las entidades territoriales para ofrecer ayuda a los consumidores de tabaco. Sin embargo, en esta medida el país actualmente se encuentra en fase de implementación y una de las pocas experiencias que existen es la Clínica para dejar de fumar, a cargo de la Pontifica Universidad Javeriana (38). Esta cuenta con un equipo interdisciplinario (médicos familiares, psicólogos clínicos, enfermeros) que realiza intervenciones que se desarrollan en cuatro etapas:

1. Valoración de la intensidad del consumo de cigarrillo y el grado de motivación para dejar de fumar.

2. Determinación del plan de intervención.

3. Establecimiento de intervención definitiva (posterior a la presentación del resultado de la evaluación interdisciplinaria al paciente).

4. Sesiones periódicas de seguimiento.

\section{Conclusión}

— n términos generales, Colombia ha realizado esfuerzos apropiados para la aplicación del Convenio Marco de la oms para el Control del Tabaco; prueba de esta labor es la aplicación aquí mencionada del Plan de Medidas MPOWER en tres ámbitos (políticas públicas, comunitario y servicios de salud) del Plan Decenal para el Control del Cáncer (PDCC) 2012-2021.

El aumento del tamaño de las advertencias sanitarias y el análisis de su impacto en la reducción de la prevalencia de consumo de tabaco y los costos en términos de salud; la 
realización de encuestas que vigilan la prevalencia del tabaquismo en la población colombiana; y el aumento de impuestos a los productos de tabaco y el análisis para determinar su impacto sobre el comercio ilícito se destacan como las acciones más relevantes en el ámbito de políticas públicas. Sin embargo, Colombia debe avanzar hacia la reglamentación de la venta de productos de tabaco en empaquetado neutro con advertencias sanitarias fuertes; generar información sobre asuntos del tabaquismo que las encuestas mencionadas no consideran; elevar aún más los impuestos sobre los productos de tabaco; e incorporar el Protocolo para la Eliminación del Comercio Ilícito de Productos de Tabaco al ordenamiento jurídico colombiano.

En el ámbito comunitario también hay esfuerzos importantes, tales como la prohibición de todas las formas publicidad de productos de tabaco; las campañas de comunicación del Instituto Nacional de Cancerología y del Ministerio de Salud y Protección Social; así como la reglamentación de ambientes $100 \%$ libres de humo de tabaco. Al respecto, el Gobierno nacional debe realizar más campañas de comunicación y aumentar el cumplimiento de los artículos de la Ley Antitabaco que reglamentan los ambientes $100 \%$ libres de humo de tabaco.

Por último, en el ámbito de los servicios de salud, se destacan el Programa para la Cesación del Consumo de Tabaco y Atención del Tabaquismo del Ministerio de Salud y Protección Social, las acciones realizadas por la clínica de cesación tabáquica de la Pontificia Universidad Javeriana y la guía de cesación publicada por el Instituto Nacional de Cancerología. Al respecto, es necesario generar información que permita identificar si esas iniciativas producen resultados positivos, especialmente, el Programa para la Cesación del Consumo de Tabaco y Atención del Tabaquismo, ya que es la principal respuesta para brindar ayuda a la población fumadora.

Además de tener en cuenta las recomendaciones mencionadas en cada uno de los ámbitos, también es necesario considerar el uso de los sistemas electrónicos de administración de nicotina y los sistemas similares sin nicotina (SEAN/SSSN), conocidos popularmente como "cigarrillos electrónicos". De hecho, la oms hizo un llamado a regular esos dispositivos, ya que no se consideran productos libres de riesgo para la salud (1).

La evidencia científica que respalda el uso de estos sistemas como alternativa para dejar el tabaco convencional es baja, ya que se basa en ensayos controlados aleatorizados y estudios observacionales considerados de baja certeza realizados por la industria tabacalera, por lo que pueden existir conflictos de intereses (39). En contraste, hay evidencia confiable que prueba que los cigarrillos electrónicos contienen varias sustancias nocivas, algunas de ellas cancerígenas, como el benceno, el tolueno, el etilbenceno, el xileno y los hidrocarbonos (40). En la misma línea, un estudio brinda evidencia según la cual los adolescentes que consumen aerosol de cigarrillo electrónico tienen mayor exposición a sustancias tóxicas en comparación con aquellos que no lo usan; quienes consumen únicamente cigarrillo electrónico tienen hasta tres veces más metabolitos de acrilonitrilo, acroleína, óxido de propileno, acrilamida y crotonaldehído (tóxicos compuestos orgánicos volátiles), mientras que los niveles de exposición 
tóxica de quienes fuman cigarrillos electrónicos y tradicionales pueden ser hasta tres veces el registrado que en quienes consumen únicamente cigarrillo electrónico (41).

En consecuencia, es necesario realizar estudios que analicen no solo la toxicidad de los SEAN/ sssn, sino también es importante obtener información sobre pérdidas de ingreso por muerte prematura e incapacidad con base en los años perdidos de vida saludable; los costos asumidos por el sistema de salud relacionados con la atención de enfermedades relacionadas con el uso de estos dispositivos; la efectividad de las estrategias de cesación y de las acciones de comunicación anti tabáquicas; y el impacto que producirían las siguientes acciones:

1. Gravar con impuestos la venta de todos los tipos de cigarrillos electrónicos, de manera similar a como los cigarrillos tradicionales están gravados de acuerdo con la Ley 1819 de 2016).

2. Reglamentar el uso de advertencias sanitarias.

3. Prohibir todas las formas de publicidad y promoción, principalmente.

El tercer punto es sumamente relevante en la actualidad pues, ya que no existe regulación de publicidad alguna para estos dispositivos: la industria promueve su uso como una alternativa menos dañina en lugares de fácil acceso para toda la población.

En Colombia no existe información representativa sobre el uso de estos dispositivos. Sin embargo, el III Estudio epidemiológico andino sobre el consumo de drogas en población universitaria de Colombia 2016 (42) brinda información al respecto. En este se muestra que el $16.6 \%$ de los estudiantes han usado cigarrillos electrónicos alguna vez en la vida, y dicha cifra es mayor en hombres (19.7\%), que en mujeres (13.9\%). A pesar de las indicaciones, y a pesar de la evidencia que indica que estos contienen sustancias nocivas, cada vez es más frecuente la venta libre en centros comerciales y tiendas especializadas de estos dispositivos, lo cual constituye una estrategia de la industria tabacalera para evitar la normatividad antitabaco de cada país.

Teniendo en cuenta lo anterior, el Congreso de la República ha intentado (de manera fallida) regular la venta y uso de esos dispositivos en al menos tres oportunidades por medio de los proyectos de ley 96 de 2014, 124 de 2016 y 167 de 2017 (todos ellos iniciativa de la Cámara de Representantes). Lo anterior sugiere que se requiere de mayor voluntad política, o de lo contrario la industria tabacalera continuará comercializando los SEAN/ssSN, ya que la normatividad vigente no aplica a los cigarrillos electrónicos.

Finalmente, se debe señalar que la implementación de todas las medidas indicadas en este artículo debe llevarse a cabo sin la interferencia de la industria tabacalera. De hecho, el Convenio Marco de la oms para el Control del Tabaco reconoce que la promoción de la salud pública y los intereses de la industria tabacalera son irreconciliables, por eso su artículo 5.3 ordena que los Estados tienen el deber de actuar para proteger las políticas de salud pública de control de tabaco contra los intereses comerciales y otros intereses creados por la industria 
tabacalera (1), ya que esta es la mayor barrera para la reducción de las muertes causadas por el consumo de tabaco (1). Aunque no existe evidencia abundante que explique cómo la industria tabacalera interfiere en las políticas de salud pública en Colombia, el Ministerio de Salud y Protección Social debe tomar cartas en el asunto. En ese sentido, se destaca el Resumen de evidencia para política. Opciones para contrarrestar las acciones de interferencia de la industria tabacalera en Colombia, en el cumplimiento del artículo 5.3 del Convenio Marco para el Control Del Tabaco y sus normas concordantes, en el cual se identifican las principales acciones al respecto realizadas en otros países (44). Colombia debe tomar en cuenta las lecciones aprendidas en otros contextos para mejorar la efectividad de todas las medidas implementadas para el control del consumo de tabaco.

\section{Contribución de los autores}

T uis Miguel Tellez Neira: concepción de la idea, búsqueda de literatura y elaboración de Uversión final del manuscrito. Sandra Liliana Romero-Torres: búsqueda de literatura y revisión del manuscrito. Maria Isabel Calderón Cortés: búsqueda de literatura y revisión del manuscrito.

\section{Agradecimientos}

os autores agradecen los comentarios y aportes de Giana María Henríquez Mendoza y
Diana Restrepo Mejía (médicas especialistas del Instituto Nacional de Cancerología), los cuales fueron muy importantes para escribir este artículo.

\section{Conflicto de intereses}

Ninguno declarado.

\section{Referencias}

1. World Health Organization (wно) . WHO report on the global tobacco epidemic, 2019 [internet]. 2019 [citado 2019 jul 30 ]. Disponible en: https://www.who.int/tobacco/global_report/en/ 
2. Departamento Administrativo Nacional de Estadística (DANE). Boletín Técnico Encuesta nacional de calidad de vida (ECV) 2017 [internet] 2018 [citado 2018]. Disponible en: https://www.dane.gov.co/index.php/estadisticas-por-tema/salud/calidad-de-vida-ecv/ encuesta-nacional-de-calidad-de-vida-ecv-2017

3. Instituto de Efectividad Clínica y Sanitaria (IECS), Instituto de Evaluación Técnica en Salud (IETS). Carga de enfermedad atribuible al tabaquismo en Colombia. IECS, IETS ; 2013.

4. Ministerio de Salud y la Protección Social, Instituto Nacional de Cancerología E.S.E. Plan decenal para el control de cáncer en Colombia 2012-2021 [internet]. 2012 [citado 2019 nov 19]. Disponible en:http://www.cancer.gov.co/documentos/Plandecenalparaelcontroldelcancer/ PlanDecenal_ControlCancer_2012-2021.pdf

5. Murillo R, Quintero Á, Piñeros M, Bravo MM, Cendales R, Wiesner C, et al. Modelo para el Control del Cáncer en Colombia. Nro. 1. Serie documentos técnicos INC. 1. ${ }^{a}$ Ed. Bogotá: Instituto Nacional de Cancerología; 2006.

6. Organización Mundial de la Salud (oms). Informe oms Sobre la Epidemia Mundial de Tabaquismo-Sin humo y con vida. Plan de Medidas MPOWER [Internet]. 2008 [citado 2019 nov 19]. Disponible en: http://whqlibdoc.who.int/publications/2008/9789243596280_spa.pdf

7. Colombia, Congreso de la República. Ley 1335 de 2009, "Disposiciones por medio de las cuales se previenen daños a la salud de los menores de edad, la población no fumadora y se estipulan políticas públicas para la prevención del consumo del tabaco y el abandono de la dependencia del tabaco”. (2009 jul 21).

8. Colombia, Congreso de la República. Ley 1109 de 2006, "Por medio de la cual se aprueba el 'Convenio Marco de la oms para el control del tabaco', hecho en Ginebra, el veintiuno (21) de mayo de dos mil tres (2003)” (2006 dic 27).

9. Colombia, Congreso de la República. Ley 1819 de 2016, "Por medio de la cual se adopta una Reforma Tributaria estructural, se fortalecen los mecanismos para la lucha contra la evasión y la elusión fiscal, y se dictan otras disposiciones” (2016 dic 29).

10. Alcaraz A, Hernández-Vásquez A, Mejía A, Pinzón C, Ciapponi A, Palacios A, et al. Advertencias sanitarias y empaquetado neutro: paquete de evidencia para la implementación de políticas de control del tabaquismo en Colombia. Documento técnico IEcs. N. ${ }^{\circ}$ 38. Buenos Aires: Instituto de Efectividad Clínica y Sanitaria; 2017. Disponible en: http://www.iecs.org.ar/wp-content/uploads/ETS-empaquetado-Colombia.pdf

11. Organizacion Mundial de la Salud (oms). Empaquetado neutro de los productos de tabaco Empaquetado neutro, pruebas empíricas, diseño y aplicación. Ginebra: Organización Mundial de la Salud (oms); 2017. Disponible en: https://apps.who.int/iris/bitstream/handle /10665/255271/9789243565224-spa.pdf?sequence=1

12. Departamento Administrativo Nacional de Estadística (DANE). Boletín Técnico Encuesta nacional de calidad de vida (ECV) 2016. Departamento Administrativo Nacional de Estadística (DANE); 2017. Disponible en: https://www.dane.gov.co/files/investigaciones/ condiciones_vida/calidad_vida/Boletin_Tecnico_ECV_2016.pdf

13. Rodriguez, J, Ruiz F, Peñaloza E, Eslava J, Gómez LC, Sánchez H, Amaya JL, Arenas R BY. Encuesta Nacional de Salud 2007. Resultados Nacionales [internet]. 1. ${ }^{\text { Ed. }} 2009$ [citado 2019 nov 19]. Disponible en: https://www.minsalud.gov.co/salud/Documents/Córdoba.pdf 
14. Ministerio de Salud y Protección Social (MSPS), Universidad del Valle. Encuesta Nacional de Consumo de Tabaco en Jóvenes [Internet]. [citado 2019 nov 19]. Disponible en: https:// untobaccocontrol.org/impldb/wp-content/uploads/ENTJ-Colombia-2016.pdf

15. Dirección Nacional de Estupefacientes, Ministerio de Protección Social, Oficina de las Naciones Unidas contra la Droga y el Delito (UNODC), Comisión Interamericana para el Control del Abuso de Drogas (CICAD), Embajada de los Estados Unidos en Colombia. Estudio Nacional de Consumo de Sustancias Psicoactivas en Colombia 2008 [internet]. Bogotá, D.C; 2009. Disponible en: https://www.unodc.org/documents/colombia/ Documentostecnicos/Estudio_Nal_Completo_2008.pdf

16. Oficina de las Naciones Unidas contra la Droga y el Delito (UNODC), Comisión Interamericana para el Control del Abuso de Drogas (CICAD), Embajada de los Estados Unidos en Colombia, Ministerio de Justicia y del Derecho, Ministerio de Salud y Protección Social. Estudio Nacional de Consumo de Sustancias Psicoactivas en Colombia 2013 [Internet]. Bogotá, D.C; 2013. Disponible en: https://www.unodc.org/documents/colombia/2014/Julio/ Estudio_de_Consumo_UNODC.pdf

17. Ministerio de Justicia y del Derecho, Observatorio de Drogas de Colombia, Ministerio de Salud y Protección Social, Oficina de las Naciones Unidas contra la Droga y el Delito (UNODC), Comisión Interamericana para el Control del Abuso de Drogas (CICAD), Embajada de los Estados Unidos en Colombia. Estudio nacional de consumo de sustancias psicoactivas en Colombia 2016 [Internet]. Bogotá, D.C; 2017. Disponible en: https://www.unodc. org/documents/colombia/2018/Junio/CO03142016_estudio_consumo_escolares_2016.pdf

18. Alcaldía de Bogotá - Secretaría Disitrital de Salud, Oficina de las Naciones Unidas contra la Droga y el Delito (unODC), Comisión Interamericana para el Control del Abuso de Drogas (CICAD), Organización de los Estados Americanos (OEA), Embajada de los Estados Unidos en Colombia. Estudio de consumo de sustancias psicoactivas en Bogotá D. C . Informe Final. 2009. 108 p. Disponible en: http://www.descentralizadrogas.gov.co/pdfs/ documentacion/estudios/Estudio_consumo_SPA_en_Bogota_2009.pdf

19. Alcaldía de Bogotá-Secretaría Disitrital de Salud, Oficina de las Naciones Unidas contra la Droga y el Delito (UnODC), Comisión Interamericana para el Control del Abuso de Drogas (CICAD), Organización de los Estados Americanos (OEA). Estudio de consumo de sustancias psicoactivas en Bogotá D.C. 2016 [internet]. 2016 [citado 2019 nov 19]. Disponible en: http://www.odc.gov.co/Portals/1/publicaciones/pdf/consumo/estudios/ locales/CO031052016-estudio-consumo-sustancias-psicoactivas-bogota-2016.pdf

20. James E, Saxena A, Restrepo CF, Llorente B, Ortiz AV, Uribe MV, et al. The distributional consequences of increasing tabacco taxes on Colombia's health and finances. An extended cost-eeectiveness analysis [internet]. [citado 2019 nov 19] Disponible en: https:// openknowledge.worldbank.org/bitstream/handle/10986/28598/120180-WP-P154568 -Colombia-PUBLIC.pdf?sequence=1\&isAllowed=y

21. Maldonado N, Llorente B, Iglesias RM, Escobar D. Measuring illicit cigarette trade in Colombia. Tob Control. 2018; 0:1-7. Doi: 10.1136/tobaccocontrol-2017-053980

22. Schafferer C, Yeh CY, Chen SH, Lee JM, Hsieh CJ. A simulation impact evaluation of a cigarette excise tax increase on licit and illicit cigarette consumption and tax revenue in 36 European countries. Pub Health. 2018; 162:48-57. Doi: 0.1016/j.puhe.2018.05.017 
23. Marten R, Kadandale S, Butler J, Aguayo VM, Axelrod S, Banatvala N, et al. Sugar, tobacco, and alcohol taxes to achieve the SDGs. Lancet. 2018;391(10138):2400-1. Doi: 10.1016/ S0140-6736(18)31219-4

24. Global Tobacco Economics Consortium. The health, poverty, and financial consequences of a cigarette price increase among 500 million male smokers in 13 middle income countries: compartmental model study. BMJ. 2018;361. Doi: 10.1136/bmj.k1162

25. Organización Mundial de la Salud (oms). Impuestos sobre los productos de tabaco [internet] sf. Organización Mundial de la salud. [citado 2018 dec 27]. Disponible en: https:// www.who.int/tobacco/economics/taxation/es/

26. Organización Panamericana de la Salud Paraguay. Protocolo para la Eliminación del Comercio Ilícito de Productos de Tabaco se convierte en ley internacional en 90 días [internet] 2018. OPS [citado 2019 sep 12]. Disponible en: https://www.paho.org/par/ index.php?option=com_content\&view=article\&id=2002:protocolo-para-la-eliminacion-del-comercio-ilicito-de-productos-de-tabaco-se-convierte-en-ley-internacional-en-90-dias\&Itemid=214

27. Colombia, Corte Constitucional de Colombia. Sentencia C-830/10 (2010).

28. García Marti S, Soto N, Mejía A, Pinzón C, Ciapponi A, Palacios A, et al. Prohibición de la publicidad, promoción y patrocinio del tabaco: Paquete de evidencia para la implementación de políticas de control del tabaquismo en Colombia. Documento técnico IECS N. ${ }^{\circ}$ 40. Buenos Aires: Instituto de Efectividad Clínica y Sanitaria; 2017. Disponible en: http://www.iecs.org.ar/wp-content/uploads/Publicidad-Colombia.pdf

29. Veeduría Ciudadana para el Control de Tabaco. Veeduría Ciudadana para el Control de Tabaco [internet]. 2016. Veeduría Ciuadana para el Control del Tabaco [citado 2018 oct 23]. Disponible en: http://veeduriacontroltab.wixsite.com/vcct

30. Ministerio de Salud y Protección Social. Generación más: somos más sin tabaco [internet] sf. Ministerio de saludy Protección Social [citado 2018 abr 30]. Disponible en: https:// www.minsalud.gov.co/Paginas/Generación más somos más sin tabaco.aspx

31. Instituto Nacional de Cancerología E.S.E. ¡No Fumar es la Actitud! [internet]. sf No fumar es la actitud [citado 2018 oct 23]. Disponible en: http://nofumar.cancer.gov.co/

32. Wakefield M, Flay B, Nichter M, Giovino G. Effects of anti-smoking advertising on youth smoking: a review. J Health Commun. 2003;8(3):229-47. Doi: 10.1080/10810730305686

33. Goldman L, Glantz S. Evaluation of antismoking advertising campaigns. Jama. 1998;279(10):772-777. Doi: 10.1001/jama.279.10.772

34. Colombia, Congreso de la República. Ley 1801 de 2016, "Por la cual se expide el Código Nacional de Policía y Convivencia”. (2016 jul 26).

35. Bardach A, Calderón M, Soto N, Mejía A, Pinzón C, Ciapponi A, et al. Ambientes libres de humo: paquete de evidencia para la implementación de políticas de control del tabaquismo en Colombia. Documento técnico IECS N. ${ }^{\circ} 39$. Buenos Aires: Instituto de Efectividad Clínica y Sanitaria; 2017. Disponible en: http://www.iecs.org.ar/wp-content/ uploads/ETS-ALH-Colombia.pdf

36. Salazar L, Henríquez G. Cesación tabáquica: Compilación de las recomendaciones actualizadas para Profesionales de la salud en Colombia [internet]. Bogotá, D.C; 2016. 47 p. 
Disponible en: http://www.cancer.gov.co/files/libros/archivos/ COMPILACIÓN TABACO V final-25-08.pdf

37. Ministerio de Salud y Protección Social. Programa para la Cesación del Consumo de Tabaco y Atención del Tabaquismo [internet]. 2017 [citado 2019 nov 20]. Disponible en: https://www.minsalud.gov.co/sites/rid/Lists/BibliotecaDigital/RIDE/VS/PP/ENT/programa-cesacion-tabaco.pdf

38. Becerra N, Vivas J, Cañón M, Luna C, Castaño I. Modelo de una clínica para dejar de fumar en la Pontificia Universidad Javeriana, Bogotá, Colombia. Atención Fam. 2011;18(4):8790. Doi: 10.22201/facmed.14058871p.2011.4.27607

39. El-Dib R, Suzumura E, Akl E, Gomaa H, Agarwal A, Chang Y, et al. Electronic nicotine delivery systems and/or electronic non-nicotine delivery systems for tobacco smoking cessation or reduction: a systematic review and meta-analysis. BMJ Open. 2017;7(2). Doi: 10.1136/bmjopen-2016-012680

40. Goniewicz M, Knysak J, Gawron M, Kosmider L, Sobczak A, Kurek J, et al. Levels of selected carcinogens and toxicants in vapor from electronic cigarettes. Tob Control. 2014;23(2):133-139. Doi: 10.1136/tobaccocontrol-2012-050859

41. Rubinstein ML, Delucchi K, Benowitz NL, Ramo DE. Adolescent exposure to toxic volatile organic chemicals from e-cigarettes. Pediatrics. 2018;141(4): e20173557; Doi: 10.1542/ peds.2017-3557

42. Oficina de las Naciones Unidas contra la Droga y el Delito (UNODC), Ministerio de Justicia y del Derecho. III Estudio epidemiológico andino sobre alteraciones en las funciones ejecutivas y consumo de drogas en la población universitaria de Colombia 2016. 1. Bogotá, D.C; 2017. Disponible en: https://www.unodc.org/documents/colombia/2017/Octubre/ Informe_Universitarios_Colombia.pdf

43. Peña E, Osorio D, Rivera D, Díaz M, Calderón L, Lara A. Resumen de evidencia para política. Opciones para contrarrestar las acciones de interferencia de la industria tabacalera en Colombia, en el cumplimiento del artículo 5.3 del convenio marco para el control del tabaco y sus normas concordantes [internet]. 2014. Disponible en: https:// untobaccocontrol.org/impldb/wp-content/uploads/reports/colombia_2016_annex11_options_to_prevent_ti_interference_report.pdf 